\title{
Preface to the focused issue on enhanced recovery pathways in thoracic surgery
}

It is my great pleasure and honor to guest edit and to write a preface for this focused issue. We picked eleven medical institutions in mainland China, from Shanghai in the East, to Chengdu in the West, Beijing in the North, to Guangzhou in the South. These institutions represent the top-notched hospitals that offer the best care for thoracic surgical patients in China. AME publishing company sent their staff to interview the chief of thoracic surgery in each and every institution mentioned above, and together, they form a picture of the standard of care offered by these top thoracic surgical centres in China. This is the first time that these interviews were published in an English-medium journal, the fournal of Thoracic Disease.

Besides offering a general description of each Surgical Chief and his success story, this issue also focused on their strategies in achieving the best clinical outcome following surgery. Enhanced recovery after surgery (ERAS) is a relatively new clinical concept involving an integrated, muti-modality approach to fast-track patients to recovery following surgery. After the publication of the 2005 consensus ERAS guidelines for colorectal surgical patients (1), this has been extended to other subspecialties, and professional body dedicated to like ERAS society <erassociety.org> has been formed.

Video-assisted thoracic surgery (VATS), popularized in the early 1990's, represented not only a paradigm shift in the surgical standard of care for patients with thoracic diseases, it also marked the beginning of technology-dependence in modern thoracic surgery. Its success also stimulated the development of related fields such as anesthesiology, intensive care, physiotherapy, nutrition and nursing care. The objective is to fast-track patients to recovery, shorten hospital stay to offset the rising hospital costs. While the socioeconomic situations in many cities in China may be different from that in the West, this is, without doubt, the global trend.

\section{Acknowledgements}

I would like to thank and congratulate personally each and every author featured in this volume. To be included in this issue is itself a recognition. I also would like to congratulate AME Publishing Company for initiating this project.

\section{References}

1. Fearon KC, Ljungqvist O, Von Meyenfeldt $M$, et al. Enhanced recovery after surgery: a consensus review of clinical care for patients undergoing colonic resection. Clin Nutr 2005;24:466-77.

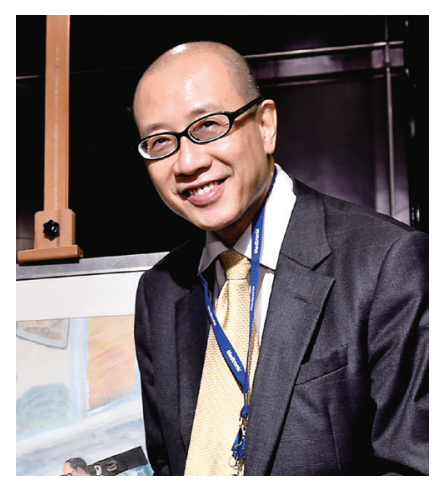

Anthony P. Yim
Anthony P. Yim Minimally Invasive Thoracic Surgery Ltd., Hong Kong, China. (Email:yimap@yahoo.com.hk) doi: $10.21037 /$ jtd.2018.05.01 Conflicts of Interest: The author has no conflicts of interest to declare. View this article at: http://dx.doi.org/10.21037/jtd.2018.05.01

Cite this article as: Yim AP. Preface to the focused issue on enhanced recovery pathways in thoracic surgery. J Thorac Dis 2018;10(Suppl 11):S1243. doi: 10.21037/jtd.2018.05.01 